$\underline{\text { Preprint typeset in JHEP style. - HYPER VERSION }}$

hep-th/0401236

\title{
Bosonic D-brane Effective Action in Linear Dilaton Background
}

\author{
by J. Klusoň \\ Department of Theoretical Physics and Astrophysics \\ Faculty of Science, Masaryk University \\ Kotlářská 2, 611 37, Brno \\ Czech Republic \\ E-mail: klu@physics.muni.cz
}

\begin{abstract}
In this paper we will study tachyon effective action for Dp-brane in bosonic string theory in the linear dilaton background. We obtain the tachyon effective Lagrangian from boundary state coeficient of Dp-brane in the linear dilaton background and compare it with tachyon effective Lagrangians that were proposed in previous papers.
\end{abstract}

KeYwords: D-branes. 


\section{Contents}

1. Introduction 1

2. Review of the tachyon condensation on D-brane in the linear dilaton background 4

3. Weak linear dilaton background 7

4. Spatial dependent tachyon profile 8

5. Conclusion 11

\section{Introduction}

Study of the tachyon condensation is one of the central themes in the string theory research in last few years [1]. Many powerful methods were developed in order to describe this process, for example it was realised that open string field theory is very useful tool for analysis of the tachyon condensation ${ }^{1}$. In some situations one can also analyse the tachyon condensation using worldsheet conformal field theory description [1, 5]. It was also shown that the tachyon condensation could be described in terms of the non-commutative geometry [6]. One of the most remarkable results given in last year is the fact that in some situation the tachyon condensation could be successfully described in terms of the effective field theory including the tachyon field and massless modes living on unstable D-brane ${ }^{2}$. Very nice discussion considering the tachyon effective field theory was given recently in [27]. According to this paper it seems that there is no much sense to find tachyon effective action only since the scale of the masses of an infinite set of massive string modes is the same at the tachyon mass and thus to keeping the tachyon while integrate out all other string modes my appear to be not well defined. On the other hand one may hope that some aspects of string dynamics can be captured by an effective field theory action invoking only the tachyon fields and massless modes, where all other massive modes decouple at a vicinity of certain conformal points. One such an example of an exact

\footnotetext{
${ }^{1}$ For reviews, see [2, 3, 䜣.

${ }^{2}$ Some papers, where the effective field theory descriptions of the tachyon dynamics can be found, are $17,8,9,10,11,12,13,14,15,16,17,18,19,20,21,23,22,24,25,26,27,28,29$.
} 
conformal point that was studied recently is a time-dependent background which should represent an exact boundary conformal theory [15, 16]

$$
T=f_{0} e^{\mu x^{0}}+\tilde{f}_{0} e^{-\mu x^{0}}
$$

where in the superstring case $\mu_{\text {super }}^{2}=\frac{1}{2}$ and in case of bosonic string theory $\mu_{\text {bose }}^{2}=1$. Its special case is the "rolling tachyon" background

$$
T=f_{0} e^{\mu x^{0}}
$$

The disk partition function in this background was recently studied in [30] suggesting that the corresponding potential term should look like

$$
V=\frac{1}{1+\frac{T^{2}}{2}}
$$

Then it was shown in [19, 29] ${ }^{3}$ that demanding that a generic first-order Lagrangian should have (1.1) (for $\mu_{\text {super }}$ ) as its exact solution fixes its time-derivative part to be

$$
\mathcal{L}=-\frac{1}{1+\frac{T^{2}}{2}} \sqrt{1+\frac{T^{2}}{2}-\left(\partial_{0} T\right)^{2}} .
$$

If we now assume as in [19] that (1.4) has a direct Lorentz-covariant generalisation we obtain the tachyon effective action for unstable Dp-brane in supersymmetric theory

$$
S=-\int d^{p+1} x \sqrt{-g} \mathcal{L}, \mathcal{L}=\frac{e^{-\Phi}}{1+\frac{T^{2}}{2}} \sqrt{1+\frac{T^{2}}{2}+g^{\mu \nu} \partial_{\mu} T \partial_{\nu} T} .
$$

The Lagrangian given in (1.5) after field redefinition $\frac{T}{\sqrt{2}}=\sinh \frac{\tilde{T}}{\sqrt{2}}$ becomes "tachyon DBI" Lagrangian

$$
\mathcal{L}_{T D B I}=-e^{-\Phi} V(\tilde{T}) \sqrt{-\operatorname{det}\left(g_{\mu \nu}+\partial_{\mu} \tilde{T} \partial_{\nu} \tilde{T}\right)}, V(\tilde{T})=\frac{1}{\cosh \frac{\tilde{T}}{\sqrt{2}}} .
$$

The arguments based on having (1.1) as an exact solution fixes only the timederivative dependence of the action (1.5). On the other hand it is possible that the covariantisation of (1.4) to (1.5) could be rather subtle [27]. In fact, the exact description for the string partition function evaluated on more general background

$$
T\left(x^{0}, x^{i}\right)=f\left(x^{0}, x^{i}\right) e^{\mu x^{0}}
$$

should be Lorentz-covariant, but that need not apply to its first-derivative part only. Exactly such a behaviour was observed in [27] where the string partition function

\footnotetext{
${ }^{3}$ For similar discussion in case of bosonic string theory see [26].
} 
in bosonic string theory was calculated on the tachyon profile $T=f\left(x^{i}\right) e^{x^{0}}$ which results into following tachyon effective Lagrangian

$$
\mathcal{L}=-\frac{1}{1+T}\left[1-\frac{\ln (1+T)}{T(1+T)}\left(\partial_{i} T\right)^{2}-\frac{s_{1}}{2(1+T)} \partial_{i}^{2} T+\ldots\right] .
$$

In supersymmetric case, where the tachyon profile is

$$
T=f\left(x^{i}\right) e^{\frac{x^{0}}{\sqrt{2}}}
$$

we obtain tachyon effective Lagrangian

$$
\mathcal{L}=-\frac{1}{1+\frac{T^{2}}{2}}\left(1+\frac{1}{1+\frac{T^{2}}{2}}\left[1-\ln \left(1+\frac{T^{2}}{2}\right)+\frac{1}{2} s_{1} \frac{1-\frac{3}{2} T^{2}}{1+\frac{T^{2}}{2}}\right]\left(\partial_{i} T\right)^{2}+\ldots\right),
$$

where $s_{1}$ can be changed by field redefinition. As was shown in [27] this Lagrangian differs from (1.5) evaluated on the profile (1.9).

The discussion given above implies that the form of the tachyon effective action strongly depends on the point in the field theory space where this action is calculated from the string partition function. For example, it was shown recently in [29] that the Lagrangian (1.5) correctly reproduces string S-matrix tree level amplitudes near the conformal point $T=e^{\frac{x^{0}}{\sqrt{2}}}$ on condition that spatial momenta of initial a final states are small. This fact gives strong support for the validity of (1.5) at least for description of tachyon dynamics close to the rolling tachyon solution (1.2). On the other hand in our recent paper [25] we have shown that there are problems when we try to apply the action (1.5) to the case of linear dilaton background. The lesson from these arguments is that we should be very careful when we try to use tachyon effective actions in regions of field theory space where its validity is not established.

In this paper we will continue the study of the tachyon effective actions in the linear dilaton background. The starting point of our analysis will be results given in very nice paper [31] where the string theory on D-branes in linear dilaton background was studied. We use the expression for the boundary state coefficient $\tilde{B}(x)$ obtained there and following the general strategy [32] we will interpret this one point function as a tachyon effective Lagrangian evaluated on the marginal tachyon profile $T=$ $\pi \lambda e^{\beta t}$. Then in order to gain some information about the tachyon effective action we will expand this one point function for case of small $T$ and also for case of "weak" dilaton background $V_{0} \ll 1$. Then we compare resulting effective Lagrangian with the tachyon effective Lagrangians obtained in previous papers [19, 27]. We find that the tachyon effective action obtained here has the similar form as the tachyon effective action for D-brane in bosonic theory (1.8). Then using the results given in [31] we will consider the case when the tachyon field is still marginal however now also depends on the spatial coordinates. We obtain an exact form of the string partition function that is manifestly Lorentz-covariant which implies that the tachyon effective 
Lagrangian evaluated on marginal tachyon profile is Lorentz-covariant as well. Since the form of this effective Lagrangian is rather complicated we restrict ourselves to the case of weak linear dilaton background. Once again we obtain action that coincides with the action given in [27.

This paper is organised as follows. In section (2) we review results given in 31. that are needed for our calculation. In section (3) we will consider the situation when the time-like component of dilaton vector is small $V_{0} \ll 1$ and we can perform an expansion of the one point function with respect to the small parameter $V_{0}$. In section (4) we generalise the calculation given in [31] to the tachyon boundary perturbation that depends on the spatial coordinates as well. In conclusion (5) we outline our results and suggest possible extension of our work.

\section{Review of the tachyon condensation on D-brane in the lin- ear dilaton background}

In this section we briefly review the description of the tachyon condensation in the linear dilaton background how was presented in [31]. We begin with the fact that tachyon condensate on unstable D-brane that describes its time-dependent decay is $T=\pi \lambda e^{\beta X^{0}}$ with $\beta>0$. We will study this process in arbitrary spacelike dilaton background $^{4}$

$$
\Phi=V_{\mu} X^{\mu}, V_{\mu} V^{\mu}>0 .
$$

The weight of the worldsheet boundary interaction operator is $\beta\left(\beta-V_{0}\right)$. Requiring the weight to be equal to 1 we obtain

$$
V_{0}=\beta-\frac{1}{\beta}
$$

As was shown in 31 the time-like part of the worldsheet CFT is the Timelike Boundary Liouville theory 33

$$
-\frac{1}{2 \pi} \int_{\Sigma}\left(\partial X^{0} \bar{\partial} X^{0}-\frac{V_{0} X^{0} R}{4}\right)+\frac{1}{2 \pi} \int_{\partial \Sigma}\left(\pi \lambda e^{\beta X^{0}}+V_{0} X^{0} K\right),
$$

where $K$ is the extrinsic curvature which integrates to $2 \pi$ around the boundary of the disc. The action (2.3) is related to the standard boundary Liouville theory with $Q=b+\frac{1}{b}$

$$
\frac{1}{2 \pi} \int_{\Sigma}\left(\partial \phi \bar{\partial} \phi+\frac{Q \phi R}{4}\right)+\frac{1}{2 \pi} \int_{\partial \Sigma}\left(\pi \lambda e^{b \phi}+Q \phi K\right),
$$

by analytic continuation $X^{0} \rightarrow i \phi, \beta \rightarrow-i b, V_{0} \rightarrow-i Q$. The boundary Liouville theory (2.4) was studied in 34, 35, 36, 37, 38, 39.

\footnotetext{
${ }^{4}$ Our convention is $\eta_{\mu \nu}=(-1,1, \ldots, p), \mu, \nu=0, \ldots, p, i, j=1, \ldots, p$. The indices in target spacetime are labelled with $I, J=0, \ldots, D-1$.
} 
Among many interesting results that were calculated in 31 the most important for our calculation is the boundary state coefficient $\tilde{B}(x)$ that is given by a disc worldsheet one point function

$$
\tilde{B}(x)=e^{V x} \int \frac{d^{D} k}{(2 \pi)^{D}} e^{-i k x}\left\langle e^{-i k X+V X}\right\rangle,
$$

where $D$ means the dimension of target spacetime. Recall that there is following relation between $D$ and $V_{\mu}$ in critical string theory

$$
V_{\mu} V^{\mu}=\frac{26-D}{6}
$$

As in [31] we require the dilaton gradient to point along the unstable D-brane.

Now we will argue how (2.5) is related to the tachyon effective Lagrangian evaluated on the profile $T=\pi \lambda e^{\beta x^{0}}$. The general definition of the stress energy tensor is

$$
T_{I J}=-\frac{2}{\sqrt{-g}} \frac{\delta S}{\delta g^{I J}}
$$

If we now presume that the tachyon effective action has the form

$$
S=-\int d^{p+1} x \sqrt{-g} \mathcal{L}
$$

we get

$$
T_{I J}(x)=-g_{I J}(x) \mathcal{L}(x)+2 \frac{\delta \mathcal{L}(x)}{\delta g^{I J}(x)} .
$$

On the other hand it was shown in 31 that the stress energy tensor of D-brane in the linear dilaton background can be expressed in terms of the boundary states coefficients $\tilde{A}_{\mu \nu}(x), \tilde{B}(x)$ as

$$
T_{\mu \nu}(x)=e^{-2 V x}\left(\tilde{A}_{\mu \nu}(x)-\tilde{B}(x) \eta_{\mu \nu}\right),
$$

where the explicit form of $\tilde{A}_{\mu \nu}(x)$ is not important for us. Comparing (2.9) with (2.10) we can anticipate that the Lagrangian evaluated on the tachyon profile $T=\pi \lambda e^{\beta t}$ is equal to

$$
\mathcal{L}(x)=e^{-2 V x} \tilde{B}(x)
$$

The expression (2.5) was calculated in [33] with the result

$$
\tilde{B}\left(x^{0}\right)=\frac{e^{\Phi}}{\beta} \int \frac{d^{D-p} k}{(2 \pi)^{D-p}} e^{i k x} \tilde{\lambda}^{i k_{0} / \beta} \Gamma\left(-i k_{0} / \beta\right) \Gamma\left(1+i \beta k_{0}\right),
$$

where

$$
\tilde{\lambda}=\frac{\pi \lambda}{\Gamma\left(1+\beta^{2}\right)}
$$


Following [31] we rewrite (2.12) into the form that will be suitable for our analysis

$$
\begin{array}{r}
\tilde{B}=\frac{e^{\Phi}}{\beta} \int \frac{d^{D-p-1} k e^{i \sum_{i=p+1}^{D-1} k_{i} x^{i}}}{(2 \pi)^{D-p-1}} \frac{d k_{0}}{2 \pi} e^{i k_{0} x^{0}} \tilde{\lambda}^{i k_{0} / \beta} \Gamma\left(-i k_{0} / \beta\right) \Gamma\left(1+i k_{0} \beta\right)= \\
=\frac{\delta\left(x_{T}\right)}{\beta} \int \frac{d k_{0}}{(2 \pi)} e^{i k_{0} x^{0}} \tilde{\lambda}^{i k_{0} / \beta} \int_{0}^{\infty} d q e^{-q} q^{-i k_{0} / \beta-1} \int_{0}^{\infty} d s e^{-s} s^{i k_{0} \beta}= \\
=\delta\left(x_{T}\right) \int_{0}^{\infty} d s \int d x^{\prime 0} \int \frac{d k_{0}}{(2 \pi)} e^{i k_{0}\left(x^{0}+\frac{1}{\beta} \ln \tilde{\lambda}-x^{\prime 0}+\beta \ln s\right)} e^{-e^{\beta x^{\prime 0}}-s} \\
=\delta\left(x_{T}\right) \int_{0}^{\infty} d s \int d x^{\prime 0} \delta\left(x^{0}+\frac{1}{\beta} \ln \tilde{\lambda}-x^{\prime 0}+\beta \ln s\right) e^{-e^{\beta x^{\prime 0}}-s}= \\
=\delta\left(x_{T}\right) \int_{0}^{\infty} d s \exp \left(-s-\frac{T}{\Gamma\left(1+\beta^{2}\right)} s^{\beta^{2}}\right)
\end{array}
$$

where we have introduced the tachyon field

$$
T=\pi \lambda e^{\beta x^{0}},
$$

and where $\delta\left(x_{T}\right)$ means the transverse delta function projecting onto the Dp-brane. In what follows we suppress this delta function. According to the discussion given above (2.14) implies that the tachyon effective Lagrangian evaluated on the marginal profile (2.15) in the linear dilaton background is equal to

$$
\mathcal{L}=e^{-\Phi} \int_{0}^{\infty} d s \exp \left(-s-\frac{T}{\Gamma\left(1+\beta^{2}\right)} s^{\beta^{2}}\right) .
$$

We present various checks that this Lagrangian reduces to the more familiar one in various limits of tachyon field theory space. First of all, for $t \rightarrow-\infty$ we can write

$$
\mathcal{L}=e^{-\Phi} \int_{0}^{\infty} d s e^{-s}\left(1-\frac{T}{\Gamma\left(1+\beta^{2}\right)} s^{\beta^{2}}\right)=e^{-\Phi}(1-T),
$$

using

$$
\int_{0}^{\infty} d s e^{-s}=1, \int_{0}^{\infty} d s e^{-s} s^{\beta^{2}}=\Gamma\left(\beta^{2}+1\right) .
$$

One can see that (2.17) is equal to the bosonic version of the Lagrangian (1.4) given in (3.5) and to (1.8) evaluated on time-dependent tachyon profile in the limit of small $T$. Moreover, for $V_{\mu}=0$ which corresponds to $\beta=1$, the effective Lagrangian (2.16) reduces to

$$
\mathcal{L}=\int_{0}^{\infty} d s e^{-s(1+T)}=\frac{1}{1+T}
$$

which is equal to the Lagrangians (3.5) and (1.8) evaluated on time dependent tachyon profile $T=e^{t}$. To gain further information about (2.16) we will consider the case when time-like component of the dilaton vector $V_{0}$ is small. Let us name this background as "weak linear dilaton background". 


\section{Weak linear dilaton background}

As we said above the formulation weak linear dilaton background means that $V_{0} \ll 1$. Since we demand that $\beta>0$ in order to describe tachyon condensation where the tachyon field rolls from $T=0$ to $T=\infty$ the condition of marginality $\beta^{2}-\beta V_{0}=1$ implies

$$
\beta=\frac{V_{0}+\sqrt{4+V_{0}^{2}}}{2} \approx 1+\frac{V_{0}}{2}, \beta^{2} \approx 1+V_{0} .
$$

Then for small $V_{0}$ we get

$$
\begin{array}{r}
\mathcal{L}=e^{-\Phi} \int_{0}^{\infty} d s \exp \left[-s-T s\left(1+(C-1) V_{0}\right) e^{V_{0} \ln s}\right]= \\
=e^{-\Phi} \int_{0}^{\infty} d s \exp [-s(1+T)]\left(1+s V_{0} T \ln \frac{1}{x}+(1-C) T V_{0} s\right)= \\
=e^{-\Phi}\left(\frac{1}{1+T}+\frac{V_{0} T}{(1+T)^{2}} \ln (1+T)\right)= \\
=\frac{e^{-\Phi}}{1+T}\left[1+\frac{\left(\beta^{2}-1\right) T}{(1+T)} \ln (1+T)\right]
\end{array}
$$

where we have used

$$
\begin{array}{r}
\Gamma\left(1+\beta^{2}\right)=\Gamma\left(2+V_{0}\right) \approx=1-(C-1) V_{0}, C=0.577, \\
\int_{0}^{\infty} d x x e^{-a x} \ln \frac{1}{x}=\frac{1}{a^{2}}(\ln a-1+C) .
\end{array}
$$

Let us say few comments about (3.2). First of all we must explain why we have replaced $V_{0}$ with $\beta^{2}-1$ using (3.1). We can argue as follows. The presence of the expression $V_{0} T$ suggests that the Lagrangian could contain terms as $\partial_{\mu} \Phi \partial_{\nu} T g^{\mu \nu}$ that are rather unusual and lead to some problems. For example, the variation of the action containing such terms with respect to $g^{\mu \nu}$ will produce expressions proportional to $\partial_{\mu} \Phi \partial_{\nu} T=\partial_{\mu} \Phi \partial_{0} T$. These expressions would be presented in the components $T_{i 0}$ of the stress energy tensor. On the other hand we know from [31] that the stress energy tensor for the rolling tachyon solution in the linear dilaton background is diagonal so that $T_{0 i}=0$. Since the Lagrangian (3.2) is evaluated on the marginal tachyon profile where (3.1) holds we mean that it is appropriate to replace $V_{0}$ with $\beta^{2}-1$. The next issue is how to interpret the expression $\left(\beta^{2}-1\right) T$. It can be written either $\frac{\dot{T}^{2}-T^{2}}{T}$ or as $\ddot{T}-T$. The first possibility is non-analytic around the point $T=0$ but together with the factor $\ln (1+T) \sim T$ for $T \ll 1$ we get analytic expression. On the other hand we mean that it is reasonable to replace $\left(\beta^{2}-1\right) T$ with $\ddot{T}-T$ in terms where the choice $\frac{\dot{T}^{2}-T^{2}}{T}$ would lead to the non-analytic behaviour around the point $T=0$. In fact, the importance of higher derivative terms in the tachyon effective Lagrangian was discussed recently in [27]. On the other hand it is 
clear that the expression $\left(\beta^{2}-1\right) T$ is in principle ambiguous and that the requirement of the analycity of the tachyon effective Lagrangian around the point $T=0$ need not be fundamental. Moreover it is possible that non-analytic terms could play significant role in some situations. We mean that in order to resolve this issue we should calculate the string partition function on more general tachyon background. However calculation such a partition function on general tachyon background is very complicated task which is certainly beyond the scope of this paper. For that reason we will follow the arguments given above so that we write (3.2) as

$$
\mathcal{L}=\frac{e^{-\Phi}}{1+T}\left(1+\frac{\ln (1+T)\left(\dot{T}^{2}-T^{2}\right)}{T(1+T)}+\ldots\right)
$$

We see that (3.4) has similar form as the Lagrangian (1.8). We must also mention that in some sense the similarity between (3.4) and (1.8) should not come as a big surprise since both Lagrangians are calculated from the string partition function even if closed string background and hence worldsheet conformal field theories are different. The more interesting question is whether there is a relation between (3.4) and the bosonic version of (1.4) given in [25] ${ }^{5}$

$$
S=-\int d^{p+1} x \frac{e^{-\Phi}}{1+T} \sqrt{1+T-\frac{\left(\partial_{0} T\right)^{2}}{T}},
$$

where we now presume that (3.5) is valid in the nontrivial dilaton background as well. Since for the weak linear dilaton the factor $T-\frac{\left(\partial_{0} T\right)^{2}}{T}=T\left(1-\beta^{2}\right)=-V_{0} T$ is small we can write ${ }^{6}$

$$
\mathcal{L}=\frac{e^{-\Phi}}{1+T}\left(1+\frac{T}{2}-\frac{\left(\partial_{0} T\right)^{2}}{2 T}\right)
$$

that is clearly different from (3.4). We mean that this fact is in agreement with the claims given in [27] that the tachyon effective Lagrangians (1.4), (3.5) do not follow directly from the string partition function. We return to this issue in the conclusion.

\section{Spatial dependent tachyon profile}

In this section we will consider the tachyon boundary interaction that also depends on spatial coordinates. More precisely, we presume that the marginal interaction inserted on the boundary of the worldsheet is

$$
T=\pi \lambda e^{\beta_{\mu} x^{\mu}},-\eta^{\mu \nu} \beta_{\mu} \beta_{\nu}+\eta^{\mu \nu} \beta_{\mu} V_{\nu}=1 .
$$

\footnotetext{
${ }^{5}$ For precise discussion of the bosonic D-brane tachyon effective action, see also 26$]$.

${ }^{6}$ It is important that (3.5) also implies $1-\left(\beta^{2}-1\right) T>1 \Rightarrow T<\frac{1}{\beta^{2}-1}$. On the other hand for exponential form of $T \approx e^{\beta x^{0}}$ there will be certainly time $x_{*}^{0}$ for which $T\left(x_{*}^{0}\right)=\frac{1}{\beta^{2}-1}$. For this time event the Lagrangian is equal to zero and then becomes imaginary. This fact again suggests problems with the generalisation of (3.5) to the linear dilaton background. We can also observe that the same problem arises in the supersymmetric version (1.4) as well.
} 
In this case the action for the worldsheet CFT has the form

$$
S=\frac{1}{2 \pi} \int_{\Sigma}\left(\eta_{\mu \nu} \partial X^{\mu} \bar{\partial} X^{\nu}+\frac{V_{\mu} X^{\mu}}{4} R\right)+\frac{1}{2 \pi} \int_{\partial \Sigma}\left(\pi \lambda e^{\beta_{\mu} X^{\mu}}+V_{\mu} X^{\mu} K\right) .
$$

From this explicit form of the string worldsheet action it is clear that the exact form of the string partition function is manifestly Lorentz covariant. On the other hand it is possible that the expansion of the partition function in powers of $\partial T$ will lead to the Lagrangian where the manifest Lorentz covariance is lost. This is an important issue that was extensively discussed recently in [27]. One can hope that the study the tachyon effective Lagrangian in the linear dilaton background could be helpful for addressing this problem. In fact, we will see that results given in this section suggest possible covariant extension of (1.8).

First of all, using the manifest Lorentz covariance we can bring $\beta_{\mu}$ in (4.1) into the frame where $T=\exp \left(\beta_{\mu} X^{\mu}\right)=\exp \left(\beta_{0}^{\prime} X^{0^{\prime}}\right)$. The the worldsheet action (4.2) is

$$
S=\frac{1}{2 \pi} \int_{\Sigma}\left(\eta_{\mu \nu} \partial X^{\prime \mu} \bar{\partial} X^{\prime \nu}+\frac{V_{\mu}^{\prime} X^{\prime \mu}}{4} R\right)+\frac{1}{2 \pi} \int_{\partial \Sigma}\left(\pi \lambda e^{\beta_{0}^{\prime} X^{\prime 0}}+V_{\mu}^{\prime} X^{\prime \mu} K\right)
$$

that has the same form as the worldsheet action given in section (2). Hence the coefficient $\tilde{B}$ will be the same

$$
\begin{gathered}
\tilde{B}\left(x^{\prime}\right)=e^{V_{\mu}^{\prime} x^{\prime}} \int_{0}^{\infty} d s \exp \left(-s-\frac{\pi \lambda e^{\beta^{\prime} x^{\prime 0}}}{\Gamma\left(1+\beta^{\prime 2}\right)} s^{\beta^{\prime 2}}\right)= \\
=e^{V_{\mu} x^{\mu}} \int_{0}^{\infty} d s \exp \left(-s-\frac{T}{\Gamma\left(1-\eta^{\mu \nu} \beta_{\mu} \beta_{\nu}\right)} s^{-\eta^{\mu \nu} \beta_{\mu} \beta_{\nu}}\right),
\end{gathered}
$$

where we have introduced the tachyon field

$$
T=\pi \lambda e^{\beta_{\mu} x^{\mu}}
$$

and we have also used explicit Lorentz covariance of $\tilde{B}$. Using the relation between $\tilde{B}$ and $\mathcal{L}$ we obtain the tachyon effective Lagrangian evaluated on the tachyon profile (4.5) in the form

$$
\mathcal{L}=e^{-V_{\mu} x^{\mu}} \int_{0}^{\infty} d s \exp \left(-s-\frac{T}{\Gamma\left(1-\eta^{\mu \nu} \beta_{\mu} \beta_{\nu}\right)} s^{-\eta^{\mu \nu} \beta_{\mu} \beta_{\nu}}\right)
$$

In order to obtain more transparent form of the Lagrangian let us again consider the case when the dilaton gradient is small. Now the condition of marginality implies

$$
-\eta^{\mu \nu} \beta_{\mu} \beta_{\nu}=1-\eta^{\mu \nu} V_{\mu} \beta_{\nu}=1+\epsilon, \epsilon \ll 1
$$


on condition of the weak dilaton background $V_{\mu} \ll 1, V_{\mu} V^{\mu}>0$. With this assumption we immediately get

$$
\begin{array}{r}
\mathcal{L}=e^{-V_{\mu} x^{\mu}} \int_{0}^{\infty} d s \exp \left(-s-\frac{T}{\Gamma\left(1-\eta^{\mu \nu} \beta_{\mu} \beta_{\nu}\right)} s^{-\eta^{\mu \nu} \beta_{\mu} \beta_{\nu}}\right)= \\
=e^{-\Phi} \int d s e^{-s(1+T)}(1-T \epsilon s \ln s+(1-C) T s \epsilon)= \\
=\frac{e^{-\Phi}}{1+T}\left[1+\frac{\epsilon T}{1+T} \ln (1+T)\right]= \\
\left.=\frac{e^{-\Phi}}{1+T}\left[1-\frac{\left(\eta^{\mu \nu} \beta_{\mu} \beta_{\nu}+1\right) T}{1+T} \ln (1+T)\right]\right] .
\end{array}
$$

Following discussion given in the previous section we perform the substitution $\left(\eta^{\mu \nu} \beta_{\mu} \beta_{\nu}+\right.$ 1) $T \Rightarrow \frac{\eta^{\mu \nu} \partial_{\mu} T \partial_{\nu} T+T^{2}}{T}$ in the term proportional to $\frac{\ln (1+T)}{1+T}$ so that we get

$$
\mathcal{L}=\frac{e^{-\Phi}}{1+T}\left[1-\frac{\ln (1+T)}{T(1+T)}\left(\eta^{\mu \nu} \partial_{\mu} T \partial_{\nu} T+T^{2}\right)+\ldots\right]
$$

The Lagrangian given above is the main result of this paper. Let us now presume that (4.9) is also valid for general tachyon not obeying the condition of marginality. Then we see that (4.9) could be considered as covariant version of (1.8). In fact, when we insert the tachyon field $T=f\left(x^{i}\right) e^{x^{0}}$ into the Lagrangian (4.9) we get

$$
\mathcal{L}=\frac{e^{-\Phi}}{1+T}\left[1-\frac{\ln (1+T)}{T(1+T)} \delta^{i j} \partial_{i} T \partial_{j} T+\ldots\right] .
$$

that same as the first order derivative part of the Lagrangian (1.8) ${ }^{7}$. Note also that for small $T$ (4.9) has the form

$$
\mathcal{L}=e^{-\Phi}\left[1-T-\eta^{\mu \nu} \partial_{\mu} T \partial_{\nu} T\right]
$$

We see that this Lagrangian is different from the standard bosonic tachyon Lagrangian describing the tachyon dynamics around the perturbative vacuum $T=0$

$$
\mathcal{L}=e^{-\Phi}\left[-\frac{1}{2} \eta^{\mu \nu} \partial_{\mu} T \partial_{\nu} T+\frac{1}{2} T^{2}\right]
$$

Note that the same thing was recently observed in 27] in case of the Lagrangian (1.8). Then it is not surprising that the tachyon profile $T=e^{\beta_{\mu} x^{\mu}}$ is not solution of

\footnotetext{
${ }^{7}$ To be precise, the term containing the second order derivatives of $T$ in (1.8) arises from the renormalisation procedure 27] that contains the beta function which is equal to zero for the marginal tachyon profile. For that reason it is not surprising that such term is missing in the (4.9) in the leading order expansion in $\epsilon$.
} 
the equation of motion that arises from (4.11) while is solution of the equation of motion arising from (4.12)

$$
\partial_{\mu}\left[e^{-\Phi} \eta^{\mu \nu} \partial_{\nu} T\right]+T=0
$$

for $\eta^{\mu \nu} \beta_{\mu} \beta_{\nu}-\eta^{\mu \nu} V_{\mu} \beta_{\nu}+1=0$. We can explain this puzzle as follows. In order to derive the correct equation of motion one needs to compute first the partition function for the general tachyon field. In our case we have calculated the partition function on the exact marginal perturbation so that it is possible that in the resulting Lagrangian some terms are missing which would be nonzero for general off shell tachyon profile. We also have ambiguity when we have replaced $\beta^{2}$ with either $\frac{\ddot{T}}{T}$ or with $\frac{\dot{T}^{2}}{T^{2}}$. In order to obtain more general effective Lagrangian we should consider arbitrary boundary tachyon perturbation on the string worldsheet and study this problem using the boundary string field theory [40].

\section{Conclusion}

The main goal of this paper was to study the tachyon effective action for Dp-brane in the bosonic string theory in the linear dilaton background. We have based this analysis on the known form of the boundary state coefficient given in 31] and its relation to the tachyon effective Lagrangian evaluated on the tachyon marginal profile. As we could see in section (2) this Lagrangian which is valid for general spacelike dilaton vector $V_{\mu}$ has rather unfamiliar form. However we have shown that in case of vanishing dilaton field $V_{\mu}=0$ the Lagrangian is the same as the Lagrangians calculated for exactly time dependent tachyon profile in [19, 27]. This coincidence also holds in the far past for general $V_{\mu}$. Much more informations we have got when we considered the case when the time-like component of the dilaton vector $V_{0}$ is small. Then we could introduce small parameter proportional to $V_{0}$ and perform an expansion in the tachyon Lagrangian with respect to it. Using the condition of the marginality of the tachyon profile we have got the tachyon effective Lagrangian that has similar form as the Lagrangian (1.8). This coincidence was much sharper when we have generalised the pure time dependent tachyon condensation to the case when the tachyon profile depends on spatial coordinates as well. We have argued that this can be done very easily using manifest Lorentz-covariance of the boundary state coefficient $\tilde{B}(x)$. Then we have found that the resulting effective tachyon Lagrangian has the same form as the effective tachyon Lagrangian evaluated in 27. On the other hand we have also shown that this Lagrangian is different from the Lagrangian (3.5) generalised to the case of the linear dilaton background. Then we have argued that this result is in agreement with the claim given in [27] where it was said that (1.4) and (3.5) do not directly follow from the string partition function. Of course this fact does not mean that the Lagrangian (1.4) is not suitable for descriptions of the 
tachyon condensation. Moreover, it was shown recently in [29] that (1.4) gives the excellent description of the tachyon dynamics around the point in the tachyon field space that corresponds to the rolling tachyon background. We must also mention very interesting paper [42] where it was shown that "tachyon DBI" Lagrangian (1.6) gives excellent description of the moduli space of unstable D-branes on a circle of critical radius. On the other hand we have shown recently in [25] that the application of (3.5) to the description of the tachyon condensation in the case of linear dilaton background leads to results which are different from results given in [31] ${ }^{8}$.

The extension of our calculations is obvious. We mean that it would be very interesting to perform the same analysis in the supersymmetric case as well. In order to do this we should firstly understand the time-like form of the $N=1$ super Liouville theory [43, 44, 45], following [33]. We hope to return to this problem in future.

Acknowledgement This work was supported by the Czech Ministry of Education under Contract No. 14310006.

\section{References}

[1] A. Sen, "Non-BPS states and branes in string theory," arXiv:hep-th/9904207.

[2] W. Taylor and B. Zwiebach, "D-branes, tachyons, and string field theory," arXiv:hepth/0311017.

[3] W. Taylor, "Lectures on D-branes, tachyon condensation, and string field theory," arXiv:hep-th/0301094.

[4] K. Ohmori, "A review on tachyon condensation in open string field theories," arXiv:hep-th/0102085.

[5] A. Lerda and R. Russo, "Stable non-BPS states in string theory: A pedagogical review," Int. J. Mod. Phys. A 15 (2000) 771 [arXiv:hep-th/9905006].

[6] J. A. Harvey, "Komaba lectures on noncommutative solitons and D-branes," arXiv:hep-th/0102076.

[7] A. Sen, "Supersymmetric world-volume action for non-BPS D-branes," JHEP 9910 (1999) 008 [arXiv:hep-th/9909062].

[8] M. R. Garousi, "Tachyon couplings on non-BPS D-branes and Dirac-Born-Infeld action," Nucl. Phys. B 584 (2000) 284 [arXiv:hep-th/0003122].

[9] E. A. Bergshoeff, M. de Roo, T. C. de Wit, E. Eyras and S. Panda, "T-duality and actions for non-BPS D-branes," JHEP 0005 (2000) 009 [arXiv:hep-th/0003221].

\footnotetext{
${ }^{8}$ See also [41].
} 
[10] J. Kluson, "Proposal for non-BPS D-brane action," Phys. Rev. D 62 (2000) 126003 [arXiv:hep-th/0004106].

[11] G. W. Gibbons, K. Hori and P. Yi, "String fluid from unstable D-branes," Nucl. Phys. B 596 (2001) 136 [arXiv:hep-th/0009061].

[12] J. Kluson, "D-branes from $N$ non-BPS D0-branes," JHEP 0011 (2000) 016 [arXiv:hep-th/0009189].

[13] J. A. Minahan and B. Zwiebach, "Gauge fields and fermions in tachyon effective field theories," JHEP 0102 (2001) 034 [arXiv:hep-th/0011226].

[14] G. Arutyunov, S. Frolov, S. Theisen and A. A. Tseytlin, "Tachyon condensation and universality of DBI action," JHEP 0102 (2001) 002 [arXiv:hep-th/0012080].

[15] A. Sen, "Rolling tachyon," JHEP 0204 (2002) 048 [arXiv:hep-th/0203211].

[16] A. Sen, "Field theory of tachyon matter," Mod. Phys. Lett. A 17 (2002) 1797 [arXiv:hep-th/0204143].

[17] A. Sen, "Time and tachyon," Int. J. Mod. Phys. A 18 (2003) 4869 [arXiv:hepth/0209122].

[18] A. Sen, "Dirac-Born-Infeld action on the tachyon kink and vortex," Phys. Rev. D 68 (2003) 066008 [arXiv:hep-th/0303057].

[19] D. Kutasov and V. Niarchos, "Tachyon effective actions in open string theory," Nucl. Phys. B 666 (2003) 56 [arXiv:hep-th/0304045].

[20] M. R. Garousi, "Slowly varying tachyon and tachyon potential," JHEP 0305 (2003) 058 [arXiv:hep-th/0304145].

[21] P. Brax, J. Mourad and D. A. Steer, "Tachyon kinks on non BPS D-branes," Phys. Lett. B 575 (2003) 115 [arXiv:hep-th/0304197].

[22] A. Sen, "Open and closed strings from unstable D-branes," Phys. Rev. D 68 (2003) 106003 [arXiv:hep-th/0305011].

[23] C. Kim, Y. Kim, O. K. Kwon and C. O. Lee, "Tachyon kinks on unstable Dp-branes," JHEP 0311 (2003) 034 [arXiv:hep-th/0305092].

[24] J. Kluson, "Particle production on half S-brane," arXiv:hep-th/0306002.

[25] J. Kluson, "Note on D-brane effective action in the linear dilaton background," JHEP 0311 (2003) 068 [arXiv:hep-th/0310066].

[26] M. Smedback, "On effective actions for the bosonic tachyon," JHEP 0311 (2003) 067 [arXiv:hep-th/0310138].

[27] A. Fotopoulos and A. A. Tseytlin, "On open superstring partition function in inhomogeneous rolling tachyon background,” JHEP 0312 (2003) 025 [arXiv:hep-th/0310253]. 
[28] J. Kluson, "D-brane effective actions and particle production near the beginning of the tachyon condensation," arXiv:hep-th/0312086.

[29] V. Niarchos, "Notes on tachyon effective actions and Veneziano amplitudes," arXiv:hep-th/0401066.

[30] F. Larsen, A. Naqvi and S. Terashima, "Rolling tachyons and decaying branes," JHEP 0302 (2003) 039 [arXiv:hep-th/0212248].

[31] J. L. Karczmarek, H. Liu, J. Maldacena and A. Strominger, "UV finite brane decay," JHEP 0311 (2003) 042 [arXiv:hep-th/0306132].

[32] A. A. Tseytlin, "Sigma model approach to string theory effective actions with tachyons," J. Math. Phys. 42 (2001) 2854 [arXiv:hep-th/0011033].

[33] M. Gutperle and A. Strominger, "Timelike boundary Liouville theory," Phys. Rev. D 67 (2003) 126002 [arXiv:hep-th/0301038].

[34] V. Fateev, A. B. Zamolodchikov and A. B. Zamolodchikov, Boundary Liouville field theory. I: Boundary state and boundary two-point function," arXiv:hep-th/0001012.

[35] J. Teschner, "Remarks on Liouville theory with boundary," arXiv:hep-th/0009138.

[36] A. B. Zamolodchikov and A. B. Zamolodchikov, "Liouville field theory on a pseudosphere," arXiv:hep-th/0101152.

[37] B. Ponsot and J. Teschner, "Boundary Liouville field theory: Boundary three point function," Nucl. Phys. B 622 (2002) 309 [arXiv:hep-th/0110244].

[38] V. Schomerus, "Rolling tachyons from Liouville theory," JHEP 0311 (2003) 043 [arXiv:hep-th/0306026].

[39] J. Teschner, "On boundary perturbations in Liouville theory and brane dynamics in noncritical string theories," arXiv:hep-th/0308140.

[40] E. Witten, "On background independent open string field theory," Phys. Rev. D 46 (1992) 5467 [arXiv:hep-th/9208027].

[41] K. Nagami, "Rolling tachyon with electromagnetic field in linear dilaton background," arXiv:hep-th/0312149.

[42] A. Sen, "Moduli space of unstable D-branes on a circle of critical radius," arXiv:hepth/0312003.

[43] T. Fukuda and K. Hosomichi, "Super Liouville theory with boundary," Nucl. Phys. B 635 (2002) 215 [arXiv:hep-th/0202032].

[44] P. Di Francesco and D. Kutasov, "World sheet and space-time physics in twodimensional (Super)string theory," Nucl. Phys. B 375 (1992) 119 [arXiv:hepth/9109005]. 
[45] E. Abdalla, M. C. B. Abdalla, D. Dalmazi and K. Harada, "Correlation Functions In Superliouville Theory," Phys. Rev. Lett. 68 (1992) 1641 [arXiv:hep-th/9108025]. 